\title{
RESTRICTED PARTITIONS
}

\author{
RAFAEL JAKIMCZUK
}

Received 2 June 2003 and in revised form 9 September 2003

We prove a known partitions theorem by Bell in an elementary and constructive way. Our proof yields a simple recursive method to compute the corresponding Sylvester polynomials for the partition. The previous known methods to obtain these polynomials are in general not elementary.

2000 Mathematics Subject Classification: 11P81.

1. Proof of Bell's theorem. The main purpose of this section is to prove the following theorem, originally proved by Bell in [1], by elementary methods.

THEOREM 1.1. For a fixed positive integer $n$, let $A 1, \ldots, A n$ be positive integers and let $M^{\prime}$ be their least common multiple. For a fixed integer $r^{\prime}$, the number of nonnegative solutions $X n, \ldots, X 1$ of $A n \cdot X n+\cdots+A 1 \cdot X 1=M^{\prime} K+r^{\prime}$, which we indicate by $D_{n}\left(M^{\prime} K+\right.$ $\left.r^{\prime}\right)$, is given by a polynomial in $K$, which is either the zero polynomial or a polynomial with rational coefficients of degree $n-1$.

First, we need the following known result.

LEMMA 1.2. For $N \geq 0$ and $m \geq 1, H_{m}(N)=0^{m}+1^{m}+\cdots+N^{m}$ is a polynomial in $N$ of degree $m+1$ with rational coefficients. Besides, $H_{m}(-1)=0$.

For example, we have

$$
H_{1}(N)=\frac{1}{2} N^{2}+\frac{1}{2} N, \quad H_{2}(N)=\frac{1}{3} N^{3}+\frac{1}{2} N^{2}+\frac{1}{6} N .
$$

There exist several elementary methods to obtain the polynomials $H_{m}(N)$.

We will see that $D_{n}\left(M^{\prime} K+r^{\prime}\right)$ is a polynomial as a direct consequence of Lemma 1.2.

The PROOF OF THEOREM 1.1. We are going to prove Bell's theorem by mathematical induction. The theorem is clearly true for $n=1$ since in this case, the number of solutions to the equation $A 1 \cdot X 1=A 1 \cdot K+r$ ' is given by the polynomials

$$
\begin{array}{ll}
D_{1}\left(A 1 \cdot K+r^{\prime}\right)=1 & \text { if } r^{\prime} \text { is multiple of } A 1, \\
D_{1}\left(A 1 \cdot K+r^{\prime}\right)=0 & \text { if } r^{\prime} \text { is not a multiple of } A 1 .
\end{array}
$$

Let $n \geq 1$ be a given, and assume Theorem 1.1 holds for $n-1$; we will prove it is also true for $n$. 
The equation corresponding to $n$ is

$$
A n \cdot X n+A(n-1) \cdot X(n-1)+\cdots+A 1 \cdot X 1=M^{\prime} \cdot K+r^{\prime}
$$

From the inductive hypothesis, we know the polynomials $D_{n-1}(M K+r)$ describing the number of solutions to $A(n-1) \cdot X(n-1)+\cdots+A 1 \cdot X 1=M \cdot K+r$ for all $r$, where $M$ is the least common multiple of $A(n-1), A(n-2), \ldots, A 1$.

We can write

$$
M^{\prime} \cdot K+r^{\prime}=A n(\alpha(K+c)+a)+b
$$

where $0 \leq b<A n$ and $0 \leq a<\alpha$.

Note that $M^{\prime} \cdot K+r^{\prime} \geq 0$ if and only if $K \geq-c$.

Letting the variable $X n$ run through all possible values of $n^{\prime \prime} \in\{0,1,2, \ldots, \alpha(K+c)+$ a\}, we obtain

$$
D_{n}\left(M^{\prime} K+r^{\prime}\right)=\sum_{n^{\prime \prime}=0}^{\alpha(K+c)+a} D_{n-1}\left(A n n^{\prime \prime}+b\right)
$$

In order to directly use the induction hypothesis, we need to express each of the terms $A n n^{\prime \prime}+b$ or the form $M K+r$, for suitable $K$ and $r$.

For that purpose, consider the set partition

$$
\begin{aligned}
& \{0,1, \ldots, \alpha(K+c)+a\} \\
& \quad=\bigcup_{0 \leq i \leq a}\{\alpha S+i: S=0,1, \ldots, K+c\} \cup \bigcup_{a+1 \leq i \leq \alpha-1}\{\alpha S+i: S=0,1, \ldots, K+c-1\} .
\end{aligned}
$$

Letting $\beta=M^{\prime} / M$, we have $A n \cdot \alpha=M \beta$ and, by (1.5), we obtain

$$
\begin{aligned}
D_{n}\left(M^{\prime} K+r^{\prime}\right)= & \sum_{i=0}^{a} \sum_{S=0}^{K+c} D_{n-1}(M \cdot(\beta \cdot S)+A n i+b) \\
& +\sum_{i=a+1}^{\alpha-1} \sum_{S=0}^{K+c-1} D_{n-1}(M \cdot(\beta \cdot S)+A n i+b) .
\end{aligned}
$$

Each $D_{n-1}(M \cdot(\beta \cdot S)+A n i+b)$ is, by induction hypothesis, a polynomial in $S$ of degree $n-2$ or the zero polynomial. The proof of Theorem 1.1 now follows directly from Lemma 1.2.

Note that (1.7) yields a recursive method to obtain $D_{n}$ from previous $D_{n-1}, D_{n-2}, \ldots, D_{1}$, which we will demonstrate in the next section.

2. An example of building method. We are going to determine the polynomial $D_{3}(12 K+8)$ that corresponds to the equation

$$
4 X 3+3 X 2+2 X 1=12 \cdot K+8
$$


Since $A 3=4$ and $\alpha=3$, (1.4) becomes $12 \cdot K+8=4 \cdot(3 K+2)$; therefore $a=2, b=0$, and $c=0$. Besides, $M=6$ and $\beta=2$. Therefore, (1.7) becomes

$$
\begin{aligned}
D_{3}(12 K+8) & =\sum_{i=0}^{2} \sum_{S=0}^{K} D_{2}(6(2 S)+4 i) \\
& =\sum_{S=0}^{K} D_{2}(6(2 S))+\sum_{S=0}^{K} D_{2}(6(2 S)+4)+\sum_{S=0}^{K} D_{2}(6(2 S)+8) .
\end{aligned}
$$

COMPUTATION OF $D_{2}(6 K)$ THAT CORRESPONDS TO THE EQUATION $3 X 2+2 \cdot X 1=$ $6 K$. Since $A 2=3$ and $\alpha=2$, (1.4) becomes $6 K=3(2 K)$; therefore $a=0, b=0$, and $c=0$. Besides, $M=2$ and $\beta=3$. Therefore, (1.7) becomes

$$
\begin{aligned}
D_{2}(6 K) & =\sum_{i=0}^{0} \sum_{S=0}^{K} D_{1}(2(3 S)+3 i)+\sum_{i=1}^{1} \sum_{S=0}^{K-1} D_{1}(2 \cdot(3 S)+3 i) \\
& =\sum_{S=0}^{K} D_{1}(2(3 S))+\sum_{S=0}^{K-1} D_{1}(2(3 S)+3) .
\end{aligned}
$$

Since the polynomial that corresponds to the equation $2 X 1=2 K$ is $D_{1}(2 K)=1$ and the polynomial that corresponds to the equation $2 X 1=2 K+3$ is $D_{1}(2 K+3)=0$, we obtain $D_{2}(6 K)=K+1$, and hence

$$
D_{2}(6(2 S))=2 S+1
$$

COMPUTATION OF $D_{2}(6 K+4)$ THAT CORRESPONDS TO THE EQUATION $3 \cdot X 2+2$. $X 1=6 K+4$. Since $A 2=3$ and $\alpha=2$, (1.4) becomes $6 K+4=3(2 K+1)+1$; therefore $a=1, b=1$, and $c=0$. Besides, $M=2$ and $\beta=3$. Therefore, (1.7) becomes

$$
\begin{aligned}
D_{2}(6 K+4) & =\sum_{i=0}^{1} \sum_{S=0}^{K} D_{1}(2(3 S)+3 i+1) \\
& =\sum_{S=0}^{K} D_{1}(2(3 S)+1)+\sum_{S=0}^{K} D_{1}(2(3 S)+4) .
\end{aligned}
$$

Since the polynomial that corresponds to the equation $2 X 1=2 K+1$ is $D_{1}(2 K+1)=0$ and the polynomial that corresponds to the equation $2 X 1=2 K+4$ is $D_{1}(2 K+4)=1$, we obtain $D_{2}(6 K+4)=K+1$, and hence

$$
D_{2}(6(2 S)+4)=2 S+1 .
$$

COMPUTATION OF $D_{2}(6 K+8)$ THAT CORRESPONDS TO THE EQUATION $3 X 2+2 \cdot X 1=$ $6 K+8$. Since $A 2=3$ and $\alpha=2$, (1.4) becomes $6 K+8=3(2(K+1))+2$; therefore $a=0$, $b=2$, and $c=1$. Besides, $M=2$ and $\beta=3$. Therefore, (1.7) becomes

$$
\begin{aligned}
D_{2}(6 K+8) & =\sum_{i=0}^{0} \sum_{S=0}^{K+1} D_{1}(2(3 S)+3 i+2)+\sum_{i=1}^{1} \sum_{S=0}^{K} D_{1}(2 \cdot(3 S)+3 i+2) \\
& =\sum_{S=0}^{K+1} D_{1}(2(3 S)+2)+\sum_{S=0}^{K} D_{1}(2(3 S)+5) .
\end{aligned}
$$


Since the polynomial that corresponds to the equation $2 X 1=2 K+2$ is $D_{1}(2 K+2)=1$ and the polynomial that corresponds to the equation $2 X 1=2 K+5$ is $D_{1}(2 K+5)=0$, we obtain $D_{2}(6 K+8)=K+2$, and hence

$$
D_{2}(6(2 S)+8)=2 S+2 .
$$

From (2.2), (2.4), (2.6), and (2.8), we have

$$
\begin{aligned}
D_{3}(12 K+8) & =\sum_{S=0}^{K}(2 S+1)+\sum_{S=0}^{K}(2 S+1)+\sum_{S=0}^{K}(2 S+2) \\
& =\sum_{S=0}^{K}(6 S+4)=6 H_{1}(K)+4(K+1) \\
& =6\left(\frac{1}{2} K^{2}+\frac{1}{2} K\right)+4(K+1)=3 K^{2}+7 K+4 .
\end{aligned}
$$

REMARKS 2.1. (i) The recursive method to compute $D_{n}\left(M^{\prime} K+r^{\prime}\right)$, given in this article, works well for relatively small values of $n$, but the computations get progressively worse as $n$ grows. (ii) A similar argument shows that there exist polynomials that count the number of solutions $X 1, \ldots, X n$, where all $X i>0$.

Acknowledgments. The author is very grateful to Professor George E. Andrews (Pennsylvania State University) for his encouragement, guidance, and assistance. Likewise, the author is very grateful to Professor David Goss (Ohio State University) and to Professor Gustavo Diaz Ciarlo.

\section{REFERENCES}

[1] E. T. Bell, Interpolated denumerants and Lambert series, Amer. J. Math. 65 (1943), no. 3, 382-386.

Rafael Jakimczuk: División Matemática, Universidad Nacional de Luján, 6700 Luján, Buenos Aires, Argentina

E-mail address: jakimczu@mai 1 . un1u. edu .ar 


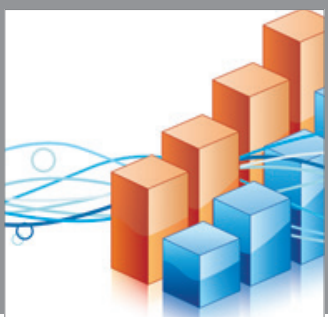

Advances in

Operations Research

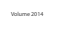

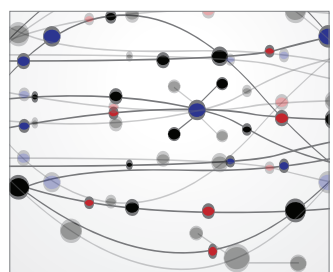

\section{The Scientific} World Journal
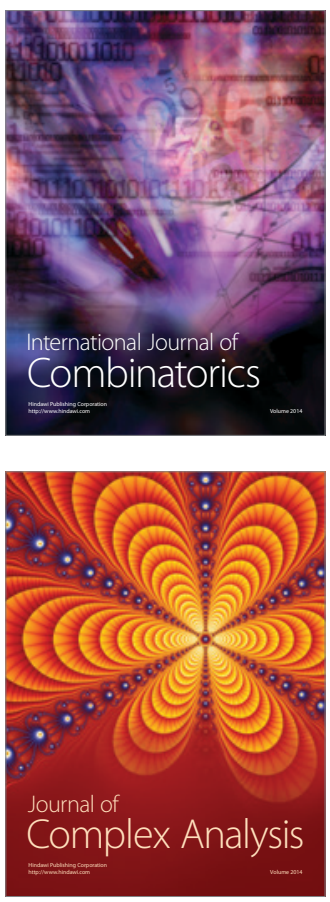

International Journal of

Mathematics and

Mathematical

Sciences
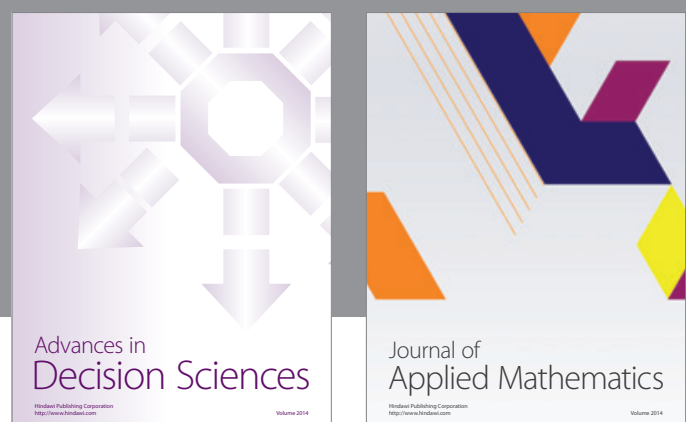

Journal of

Applied Mathematics
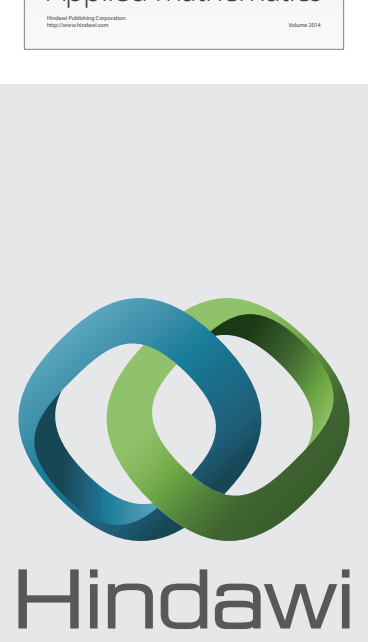

Submit your manuscripts at http://www.hindawi.com
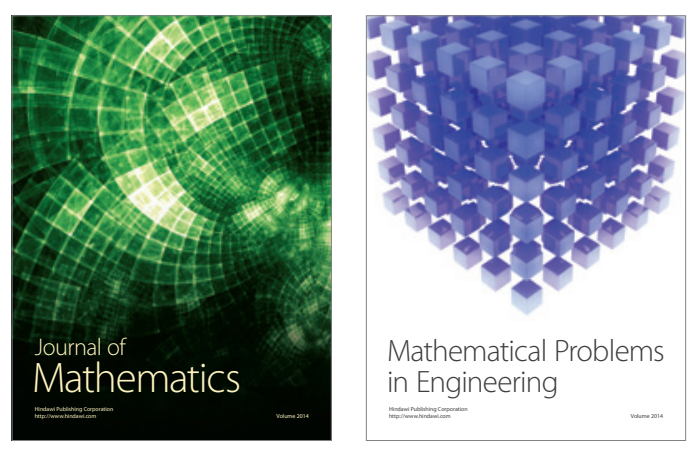

Mathematical Problems in Engineering
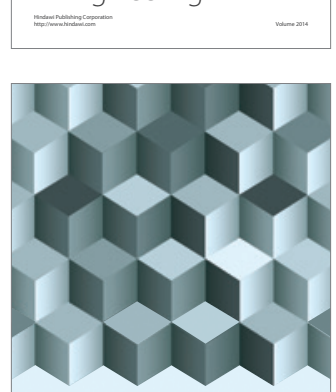

Journal of

Function Spaces
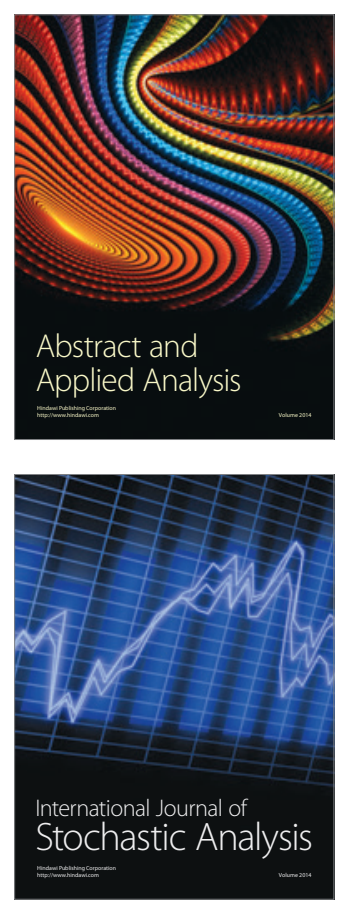

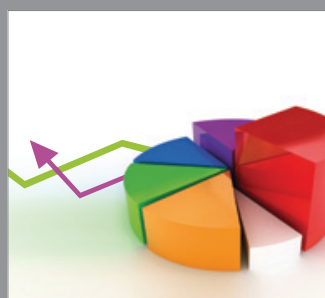

ournal of

Probability and Statistics

Promensencen
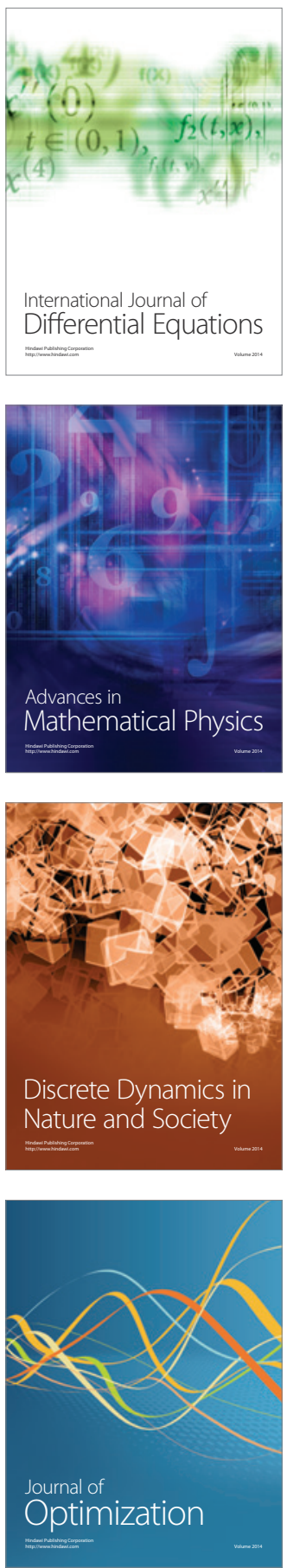